\title{
Impact of Onset Ambiguity on SEM Signature and Reduction Approach by Scattering and Polarization Diversification
}

\author{
Faisal Aldhubaib \\ Electronics Department, College of Technological Studies, Public Authority for Applied Education, Kuwait \\ Email: ff.aldhubaib@paaet.edu.kw
}

How to cite this paper: Aldhubaib, F. (2020) Impact of Onset Ambiguity on SEM Signature and Reduction Approach by Scattering and Polarization Diversification. Journal of Electromagnetic Analysis and Applications, 12, 29-42.

https://doi.org/10.4236/jemaa.2020.123004

Received: November 18, 2019

Accepted: March 27, 2020

Published: March 30, 2020

Copyright $\odot 2020$ by author(s) and Scientific Research Publishing Inc. This work is licensed under the Creative Commons Attribution International License (CC BY 4.0). http://creativecommons.org/licenses/by/4.0/

\begin{abstract}
The paper assesses how multiple-static scattering mitigates the effect of late-time onset on the robustness of the extracted resonance modes in the context of radar target classification. The assessment exploits the mode distribution vs onset shift to verify the sensitivity of the mode's extraction to the selected onset, especially higher-order, to onset. However, within some bistatic directions, the modes have enhanced energies with lesser specular energy, making the modes estimate less sensitive to onset shifts. Also, the mode distribution per bistatic and polarization configuration has demonstrated different onset windows of accurate and consistent mode extraction. Notably, the distribution of the mode energy distribution reveals that classification performance degrades with poorly selected onset.
\end{abstract}

\section{Keywords}

Bistatic Scattering, Target Classification, Polarization Scattering, Singularity Expansion Method, Feko Simulation

\section{Introduction}

A primary role of a modern radar system is to perform classification or discrimination operation of unknown targets based on a radar signal model, for which the model parameters should faithfully reflect some physical attributes of the target [1] [2]. Therefore, the robust model parameters aim to decrease the separation distance between same-class targets, and increase the separation between different classes; subsequently, obtain better classification performance curve against perturbation sources like noise. Such a signal model is the Singularity Expansion Method (SEM) that represents the late time of a pulsed signal of tran- 
sient nature as a set of multiple decaying exponentials, i.e., resonance modes, where a matrix pencil method (MPM) can extract the mode parameters [3] [4]. Then the reconstructed late-timesignalis expressed as follows:

$$
y_{\text {lrec }}(t)=\sum_{m=1}^{M} a_{m} \cdot \sin a_{m} t \cdot e^{\delta_{m} t}
$$

where $\omega \equiv$ angular resonant frequency, normalized $\hat{\delta} \equiv$ the damping factor/ wave speed, $a_{n} \equiv$ associated residue. Henceforth, the ratio $|a / \hat{\delta}|$, namely Residue/damping factor, stands for the mode energy normalized by the speed of light. The modal order parameter $M$ represents the highest order in the model, and it should reflect the number of the cardinal structures of interest that should be excited by the pulse.

The computation includes a matrix pencil method (MPM) to extract the mode parameter set, namely $(\omega, \hat{\delta}, a)$, from the selected transient signal [3] [4]. The mode has enhanced return with its frequency related to the target's cardinal dimensions. Also, a low-order mode extends temporally revealing single structure interaction, whereas, a higher-order mode localizes temporally revealing multiple structure interactions [5]. Theoretically, each class of targets possesses a unique mode set which may help discriminate the class from the clutter and interference in a high-volume radar cell.

Nevertheless, as the specular portion is adjacent and preceding the late-time has a more substantial return, it is necessary to select a late-time onset that maximizes the separation between the modes and the specular component, without omitting high-order modes. Thus, onset ambiguity when extorting the mode set is considered a significant factor that could degrade the effectiveness of the SEM model. Notably, the onset ambiguity limits the validity of the intended classification operation in a noncooperative scenario, especially when the proposed operation is over a range of ambiguous target aspects, such as with concealed targets. Hence, such SEM-based classification techniques will perform suboptimally due to the limitation imposed on the number of modes in the model when some modes are omitted by improper onset selection.

In this respect, previous studies used the Extinction pulse (E-pulse) method (introduced by Rothwell et al. [6]), but have assumed cooperative targets, i.e., target dimensions known; thus considered that the onset is simply after a period of twice the transit time to pass the most longitudinal dimension, i.e., farthest tips, of the target [7] [8] [9] [10]. As a result, the onset was considered static irrespective of the illuminated target aspect. The static onset assumption leads to spurious modes at some scattering directions and thus lowers the SEM ability to reflect fatal information about target attributes.

Henceforth, the paper hypothesizes that the mode estimate energy can improve over some incident to scattering configuration and polarization channels. Therefore, the assessment begins in the frequency domain to attain a reference (ideal) modal-regions from the magnitude peaks, and from which the twice reciprocal-frequency of the first peak gives a reference onset to benchmark in the 
time-domain analysis [11]. In the time domain, the assessment considers the MPM extracted modal-frequency distribution as a function of onset shift to reveal onset bins of well-estimated modes.

The aim is to find onset bins of robust mode extraction per the scattering direction and polarization channel. Finally, the assessment views the onset effect on the target classification by a scatter plot of the mode energy versus modal-frequency per scattering configuration and channel given three onset cases.

\section{The Simulation}

The commercial software FEKO can construct a generic aircraft target model and calculate the scattered E-field data by a method of moments solver [12]. Figure 1 depicts model $\mathrm{A}$ of the target (with dimensions annotated) with a wing leading edge swept-back by $50^{\circ}$. Then model B of the target, i.e., duet model, has the wing and tail parts swept downward by $20^{\circ}$ in the elevation plane to benchmark the discriminative ability of the SEM signature for same-class targets subject to onset uncertainty. Wedged structures dominate the proposed target class, i.e., wing, tail and stabilizer, of cardinal electrical dimensions (of half-wavelengths equivalent to 8 - $27 \mathrm{MHz}$ range.) Theoretically, the aircraft modal frequencies could extend from the fundamental region in the high-frequency (HF) band to the decade region in the very high frequency (VHF) band.

The simulation adopts a plane wave excitation of bandlimited frequency (between highest $f_{H}$ and lowest $f_{L}$ with frequency sampling rate $\Delta f \hat{f}$ and propagating in the incidence direction $\left(\hat{\beta}_{\text {inc }}\right)$ with a linear polarization direction $(\eta)$ towards the coordinate system origin. The $\hat{\beta}$ vector gives the elevation (or Theta) angle $(\mathfrak{V})\left(\right.$ from $z=0$ ) and azimuth (or Phi) angle $(\varphi)$ (from $x=0$ ); while $\eta=\left[0,90^{\circ}\right]$ represents a vertical ( $\mathrm{V}$ or Theta) direction perpendicular to the azimuth plane
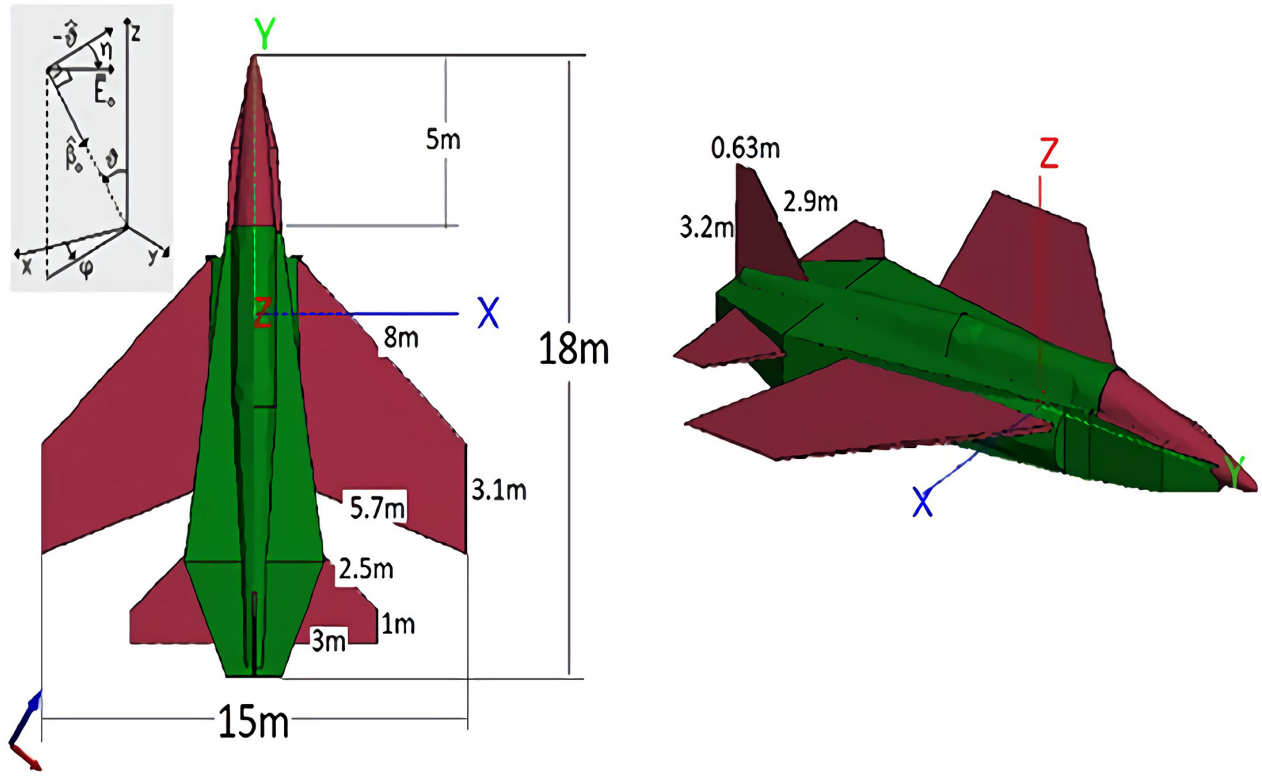

Figure 1. The aircraft's model. Main Frame made of $0.005 \mathrm{~m}$ thick Titanium (green). 
and a horizontal ( $\mathrm{H}$ or $\mathrm{Phi}$ ) direction parallel to the azimuth plane, respectively. Moreover, the simulation derives the scattering data for the modal frequencies for azimuth cut, i.e., 0-2-360 . Table 1 summarizes FEKO simulation variables. A Gaussian pulse $u(t)$ of a duration $\left(t_{d}\right)$, peak amplitude $\left(u_{o}\right)$, pulse delay $\left(t_{o}\right)$ and pulse width $\left(p_{w}\right)$, can be expressed as follows

$$
u(t)=u_{o} e^{m_{a}^{2}\left(t-t_{0}\right)^{2}}, 0 \leq t<t_{d}
$$

Here $m_{a}=\frac{2 \sqrt{\ln (2)}}{p_{w}}$, Table 2 describes the pulse parameters values with sampling time $\Delta t$ for the discrete version.

The Fourier transformation converts the coherent frequency data to a temporal data (consisting of early (specular) and late (transient) portions). The convolution with a baseband Gaussian pulse (depicted in Figure 2.) synthesises a baseband excitation necessary to excite the fundamental modes.

Table 1. Far-field calculations step up (frequency and aspect configurations).

\begin{tabular}{|c|c|c|c|}
\hline Parameter & Value & Parameter & Value \\
\hline$f_{L}$ & $0.1 \mathrm{MHz}$ & $f_{H}$ & $50 \mathrm{MHz}$ \\
\hline$N_{f}(\Delta f)$ & $64(779 \mathrm{kHz})$ & $\vartheta$ & $110^{\circ}$ (fixed) \\
\hline$\varphi_{\text {inc }}$ & $235^{\circ}$ & $\varphi_{\text {scat }}$ & $\begin{array}{c}(235,325,145,55)^{\circ} \\
(0: 2: 360)^{\circ}\end{array}$ \\
\hline
\end{tabular}

Table 2. The Gaussian pulse parameters.

\begin{tabular}{cc}
\hline Parameter & Value \\
\hline$t_{d}$ & $\approx 500 \mathrm{~ns}$ \\
$p_{W}$ & $20 \mathrm{~ns}$ \\
$\Delta t$ & $1.95 \mathrm{~ns}$ \\
\hline
\end{tabular}

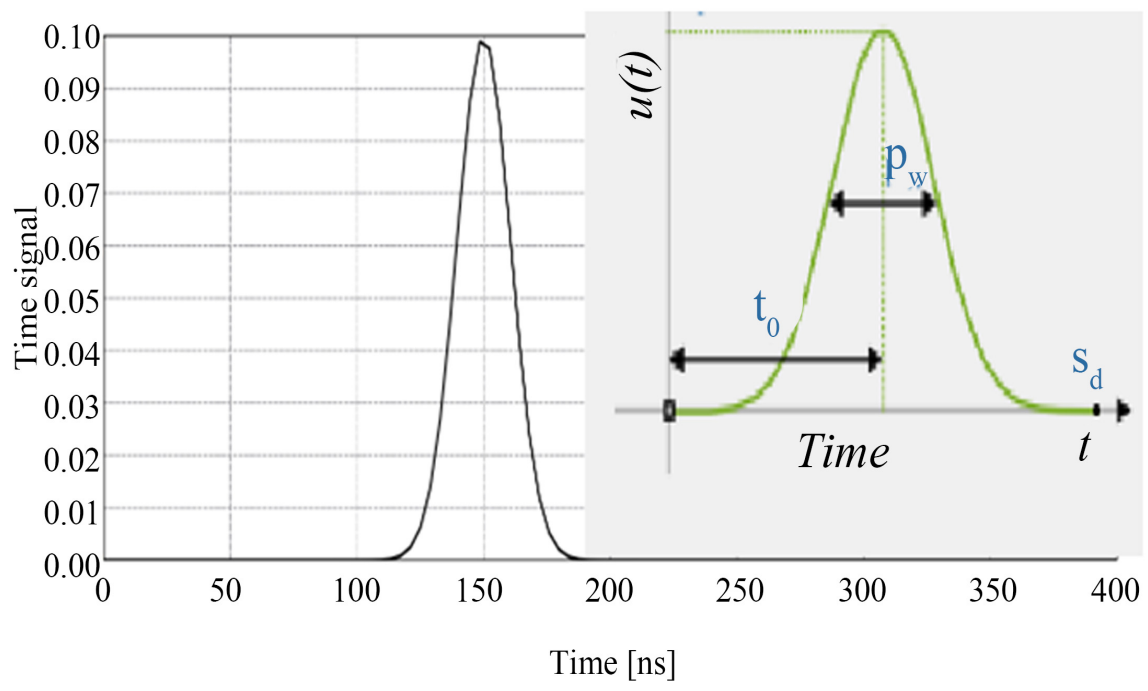

Figure 2. The incident pulse of standard Gaussian shape. 


\section{Procedures}

The following steps describe the procedures:

a) Use Feko calculate the coherent frequency response for the four orthogonal scattering direction of interest.

b) Consider that the reference frequencies as the frequencies of the peaks, then derive their azimuthal pattern to verify that the mode enhances in some scattering and polarization directions.

c) Define a reference onset as the reciprocal of the first frequency plus the delay time and pulse width (for backward scattering).

d) Use the Fourier transformation then the convolution with the Gaussian pulse to transform the coherent frequency data to time data, namely $y_{0}$, consisting of early and late time parts.

e) Truncate the whole signal by a unit step function shifted by an onset time, namely $T_{l}$, to create a truncatedsignal, namely $y_{l}$, as follows:

$$
y_{l}(t)=y_{0}(t) \cdot \delta\left(t-T_{l}\right)
$$

f) Repeatedly apply the MPM to the truncated signal while shifting the onset to derive the mode frequency distribution per polarization and scattering directions of interests.

g) Derive the distribution of the residue/damping factor versus frequency for earlier, reference and later onsets for model A per scattering direction, then for both models per polarization channel.

\section{The Result}

\subsection{Frequency Analysis}

Figure 3 depicts the simulated backscattered frequency data in the backward direction, demonstrating distinctive mode regions (peaks) that match the halfwavelengths equivalent of the target's global structures. The direction order $H H$, $V V$ and $H V$ represent the polarization directions of the excitation and reception ends, respectively. According to the target dimensions, the wings, stabilizers, and the tail theoretically resonate according to their equivalent half-wavelengths corresponding to $8.89,11.18$ and $23 \mathrm{MHz}$ and their higher orders, e.g., at 17.5 and $43 \mathrm{MHz}$, which is confirmed by the calculated far-field response. In general, the different polarization channels ( $H H$ and $V H$ ) have different dominant mode sets. The cross-polarization direction $h v$ has better localization as the specular return is less profound.

Figure 4 shows that the modes are excited by different strengths according to the scattering and polarization configuration, where the frequency return is much more substantial within the forward direction above 11 and $35 \mathrm{MHz}$ for the $H H$ channel, and the side directions for the $V H$ channel. Importantly, the first modal region at $8.9 \mathrm{MHz}$ gives a reference onset at $232 \mathrm{~ns}$.

Figure 5 depicts the magnitude of the modes within the $360^{\circ}$-azimuthal radiation pattern for $\mathcal{\vartheta}=110^{\circ}$ plane cut. Notably, the forward and sideward directions 


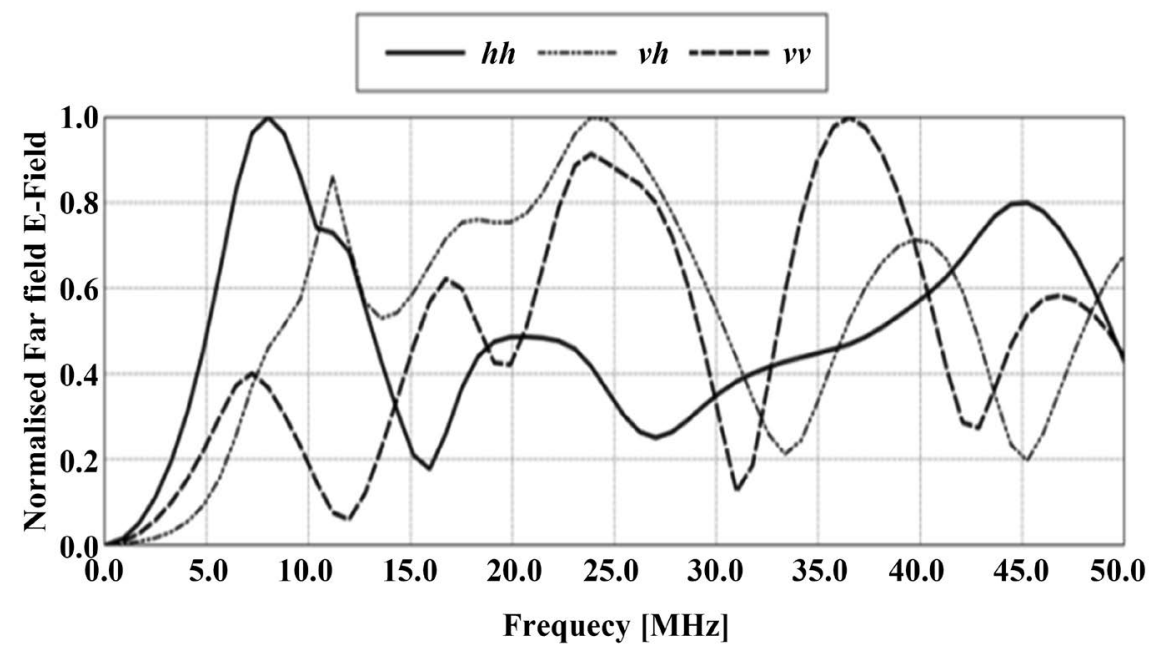

Figure 3. The calculated far-field responses within different polarization channels.

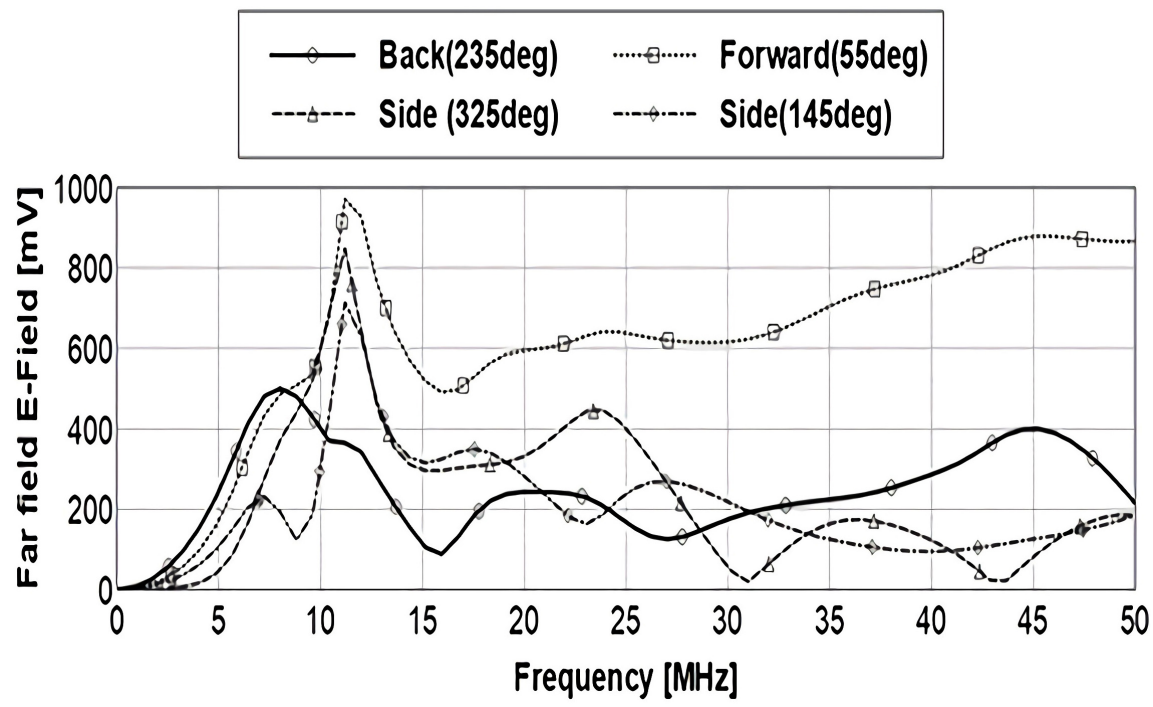

(a)

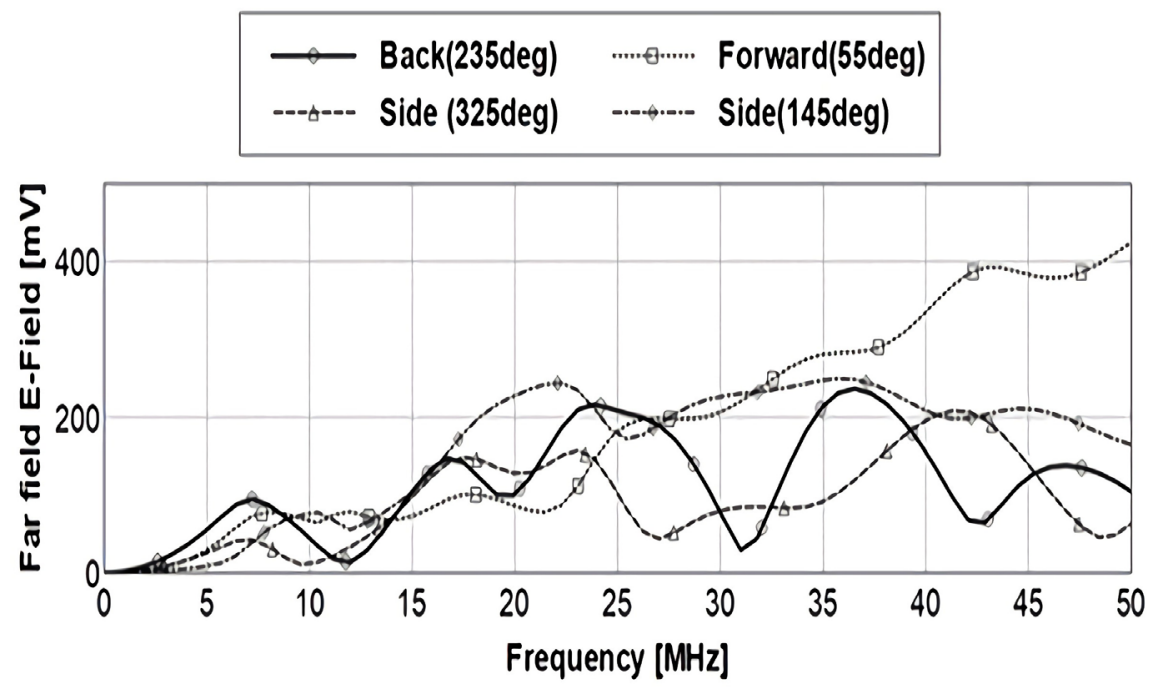

(b) 


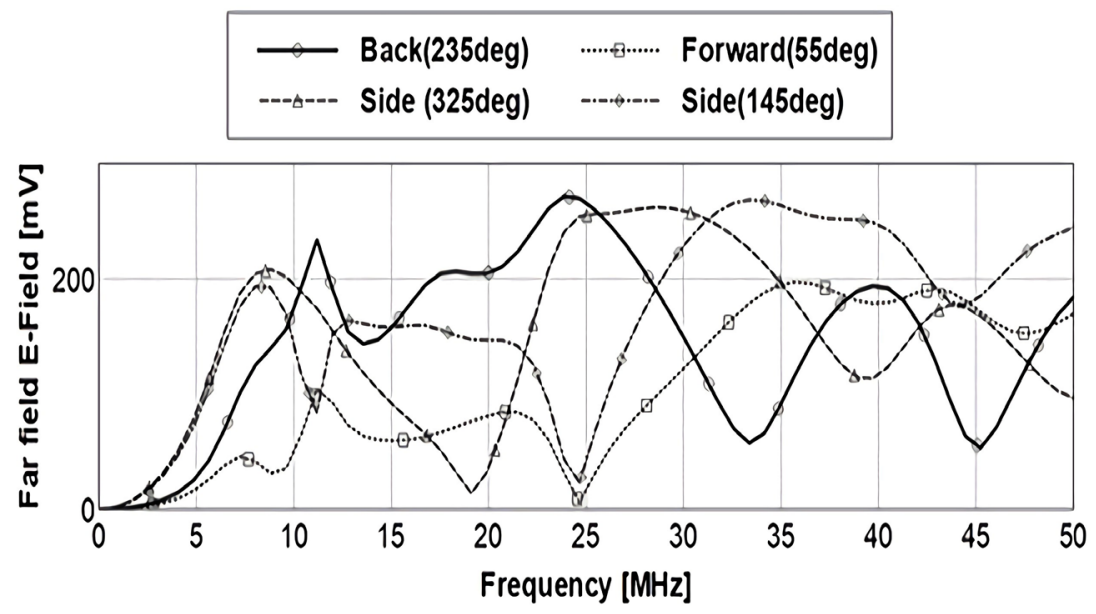

(c)

Figure 4. The calculated E-field responses for forward and side directions (with backscatter direction as a reference). (a) $H H$; (b) $V V$; (c) $V H$.

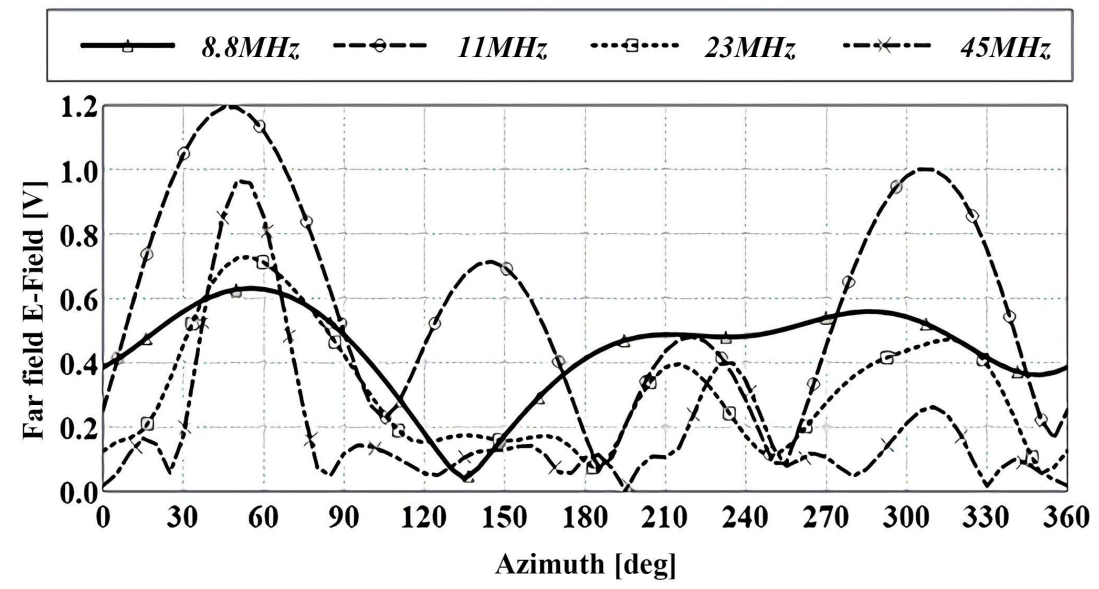

(a)

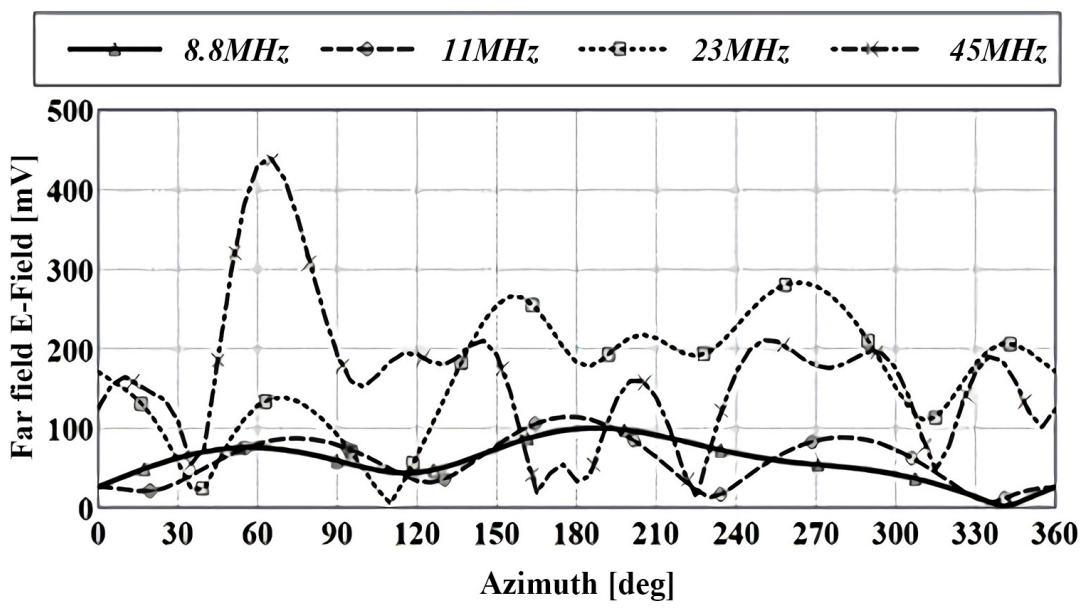

(b)

Figure 5. The azimuthal scattering pattern in $\vartheta=110^{\circ}$ per co-polarized channel. (where backward direction at 235, the forward direction at 55 and side directions at 325 and 145). (a) $H H$; (b) $V V$. 


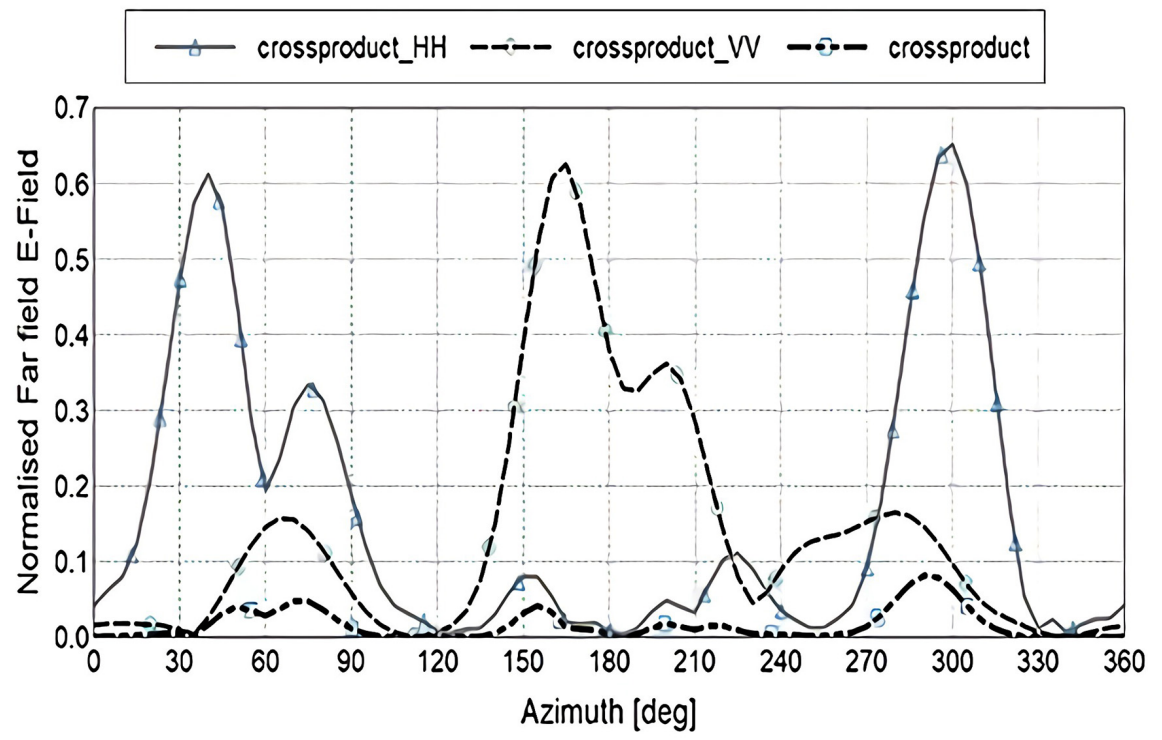

Figure 6. The azimuthal pattern of the modes cross-product.

have enhanced magnitude return compared to the backward direction, especially at the second mode where the wing and stabilizer interaction dominates. For the $H H$ channel, the modes have all enhanced magnitude near the 180 forward direction, i.e., $\varphi=55^{\circ}$. In overall, the $11 \mathrm{MHz}$ mode has the highest total return in the $H H$ channel due to the wing interaction, whereas the $23 \mathrm{MHz}$ mode has the highest total radiation in the $V V$-channel due to the tail interaction. Notably, as the higher mode reveals more interactions between the target's structures, the mode's radiation pattern becomes more directive in the forward and side directions.

Figure 6 depicts the cross-product patterns $H H$ and $V V$ of the four modes in term of the co-polarized channels, which reveals enhanced product returns for $H H$ channel near $-195^{\circ}$ and $+60^{\circ}$, and $V V$ channel near $-70^{\circ}$ offset the backward direction. Nevertheless, a single bistatic direction of enhanced performance for all four modes does not exist across both polarization channels, suggesting that the modal order should be kept minimal as possible, hence, $M=3$ is a default choice for this case of classification.

\subsection{Time Analysis}

Figure 7 depicts the pulsed scattered responses for model A in the four orthogonal scattering directions, showing that the transient response is almost 30 -fold the excitation pulse duration. In general, the transient return is extended and oscillatory with profoundly specular response existing within the backscatter direction for a period of approximately $120 \mathrm{~ns}$, while other scattering directions suppress the specular portion, but enhance the transient part.

Figure 8 depicts the distribution of the first three modes with onset shift within the backward direction in the horizontal direction. In general, the stability of the first two modes is more extended compared to the third resonance, 


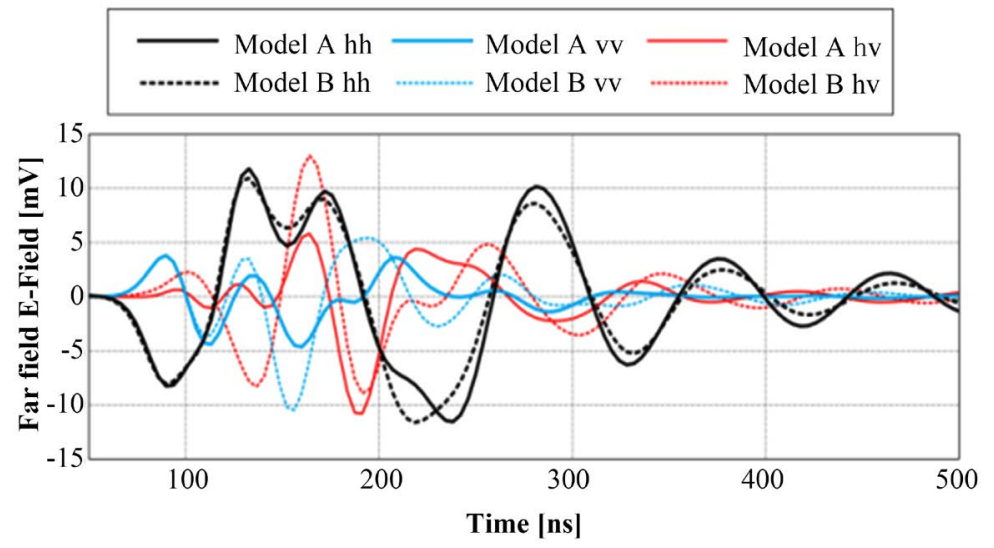

Figure 7. The transient responses per scattering direction for $h h$-pol polarization channel.

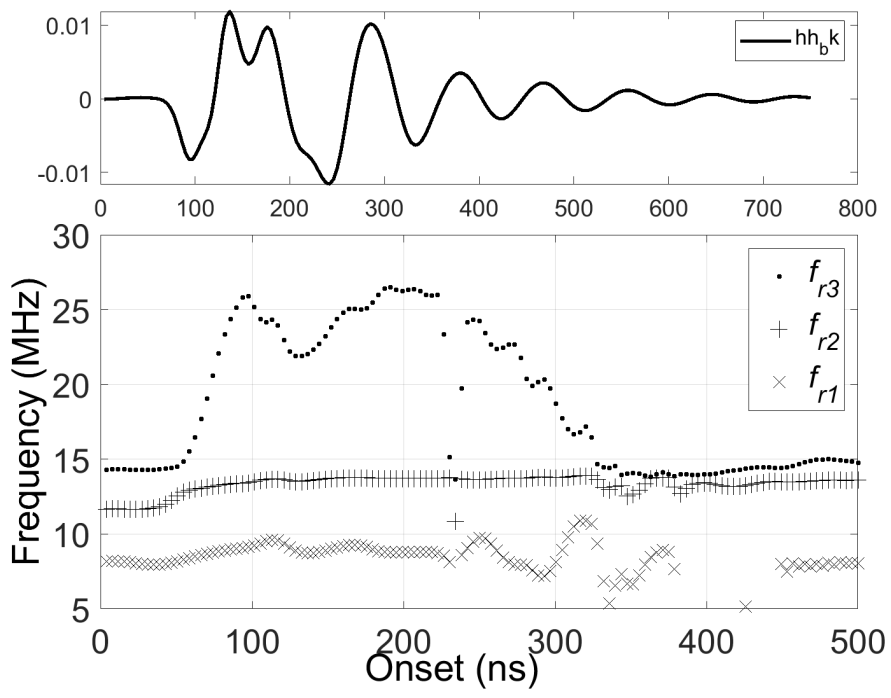

(a)

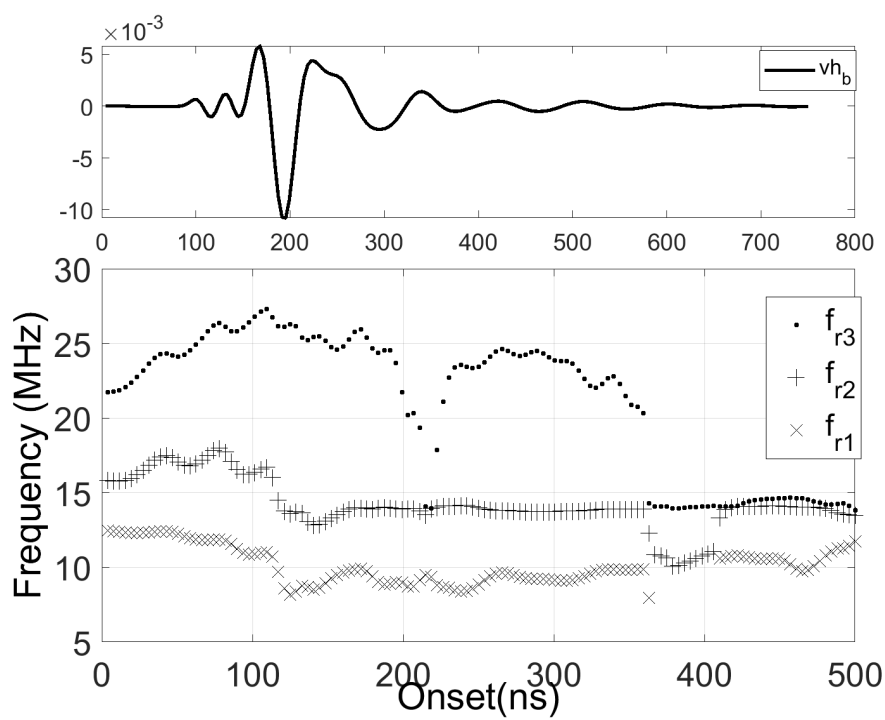

(b)

Figure 8. The distribution of three-mode frequencies with onset shift in the backward direction for co- and cross-polarized channels. (a) $h h_{b k}$; (b) $v h_{b k}$. 
which extends for about $120 \mathrm{~ns}$ between the onset interval of $100-220 \mathrm{~ns}$ within the co-polarized channel. However, the third mode extends for about $350 \mathrm{~ns}$ between the onset interval of 0 to 350 ns with better consistency within the cross-polarized channel. Notably, the onset interval between 225 - 350 ns within the cross-polarized channel demonstrates better consistency for all three modes.

Figure 9 depicts the distribution of the first three modes with onset shift within the forward directions in the $h h$ channel. The onset shift intervals of $200-300 \mathrm{~ns}$ demonstrate stable extraction of the three modes. Practically, delaying the onset interval will eventually decrease the transient energy to the specular level to the extent that the MPM cannot robustly extract the modes. Next, the dependence of the energy of the modes on the scattering direction is noticeable as depicted in Figure 10, with the backward direction having the lowest energy in the $h h$ channel case. The returns of the first and second modes fluctuate less across the different scatter directions because of their low directivity compared to the third mode, which shows more fluctuations with scattering direction. The mode set of the earlier time onset at $T_{I}=113 \mathrm{~ns}$ shows better resemblance to at $T_{I}=210 \mathrm{~ns}$ (the default), with higher energy. Contrary, the delayed time onset at $T_{I}=347 \mathrm{~ns}$ shows modes of lower energies that have less resemblance and noticeable fluctuations along the different scattering direction. In general, shifting the onset to later times causes omitting overdamped modes because they are highly localized in time, as seen in the case of the third mode in Figure 10(b), in which the modes beyond $16 \mathrm{MHz}$ disappear. Figure 11 depicts the energy distribution of the modes per polarization channel for both models. For $T_{I}=113 \mathrm{~ns}$ and $210 \mathrm{~ns}$, the modes distributions of both models are relatively similar compared to $T_{I}=$ $347 \mathrm{~ns}$; hence, proper onset selection is essential to classify a target correctly.

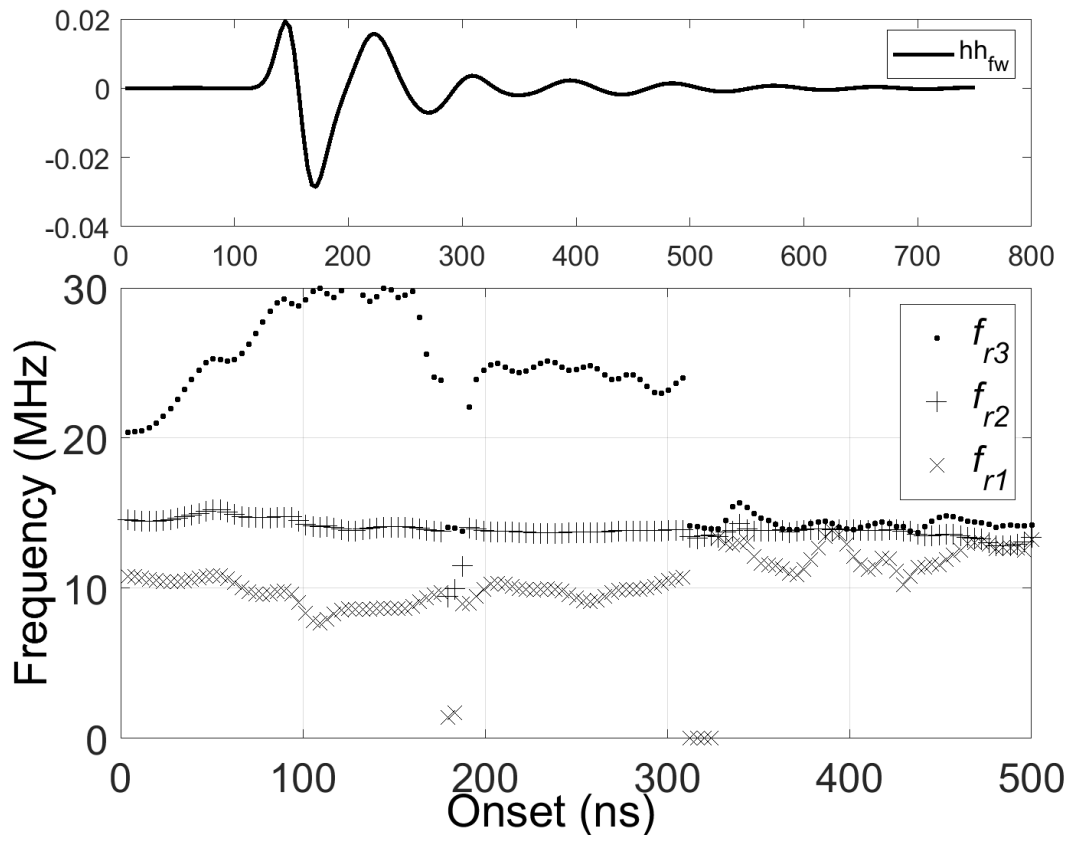

Figure 9. The distribution of three-mode frequencies with onset shift in forward direction for $h h$-pol channel. 


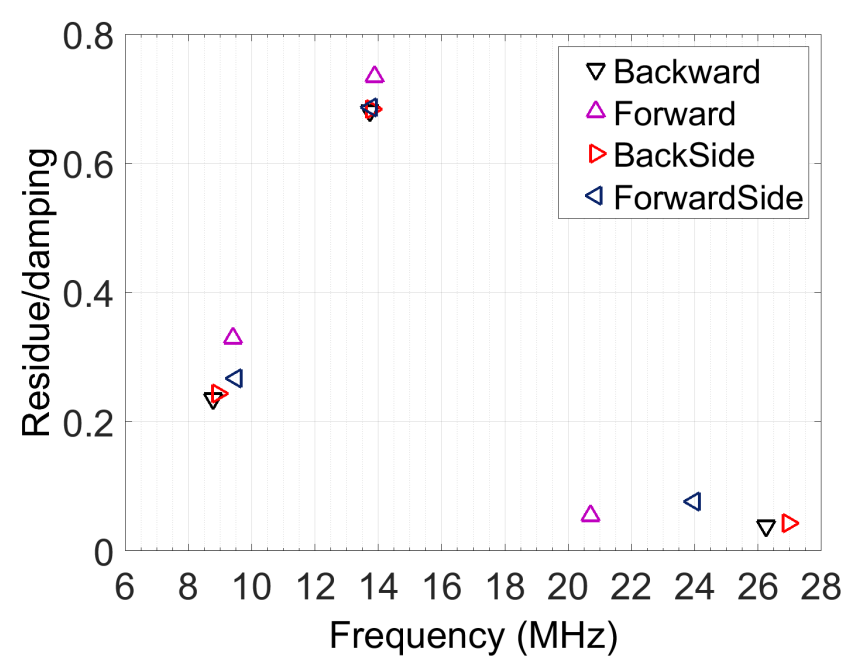

(a)

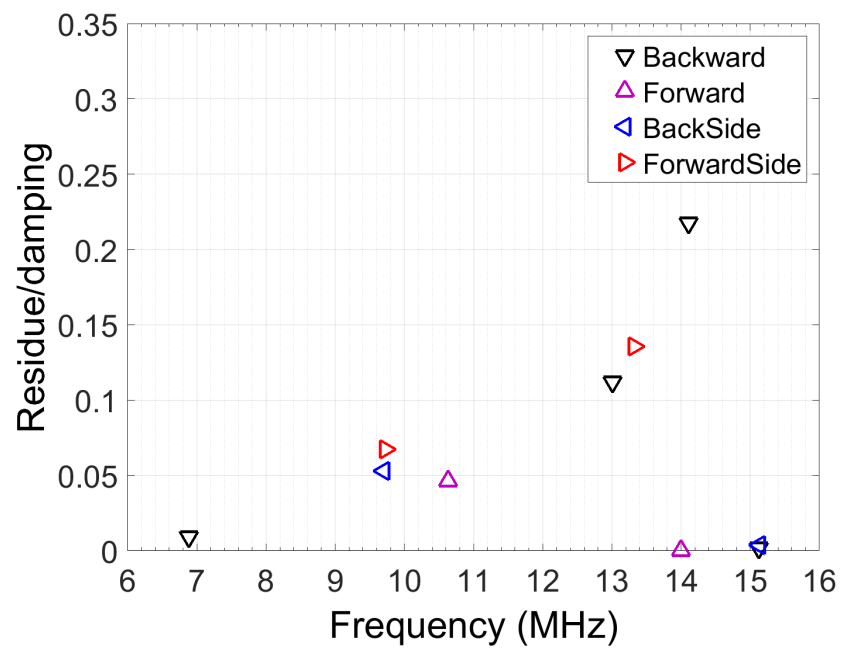

(b)

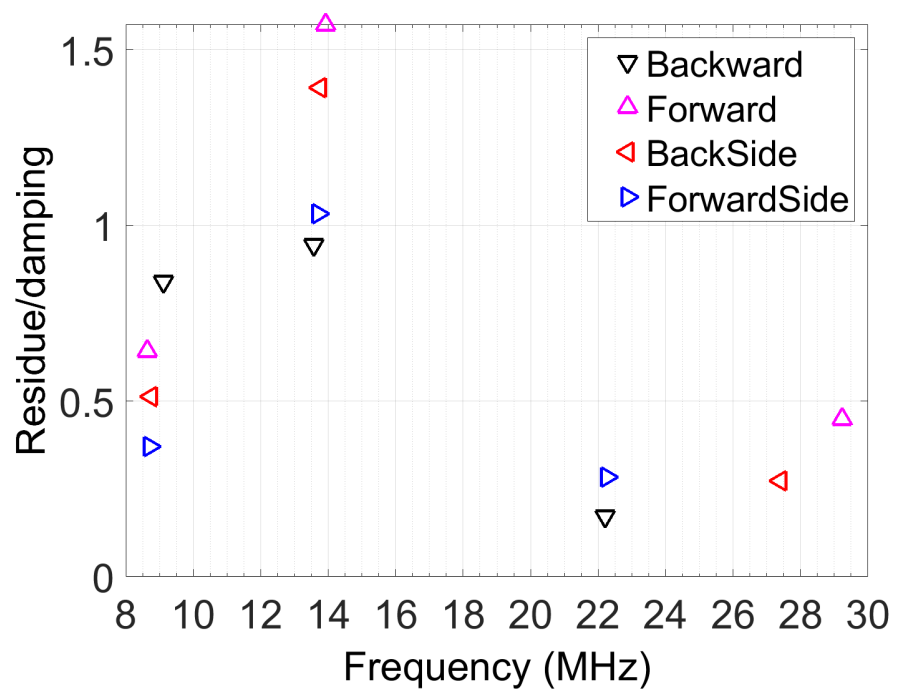

(c)

Figure 10. The $h$ h-energy distribution per scattering direction. (a) $T_{l}=225 \mathrm{~ns}$; (b) $T_{l}=$ $347 \mathrm{~ns} ;$ (c) $T_{1}=113 \mathrm{~ns}$. 


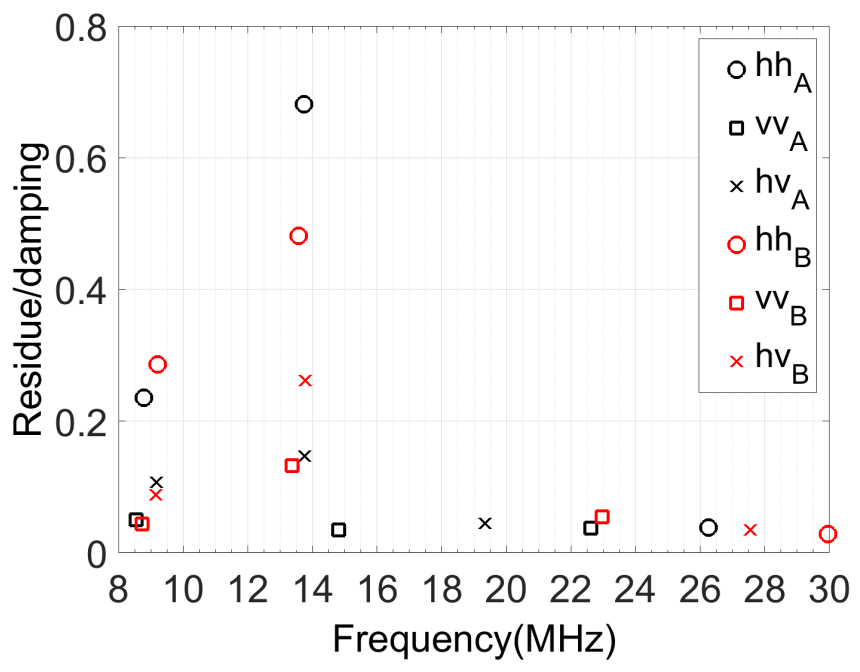

(a)

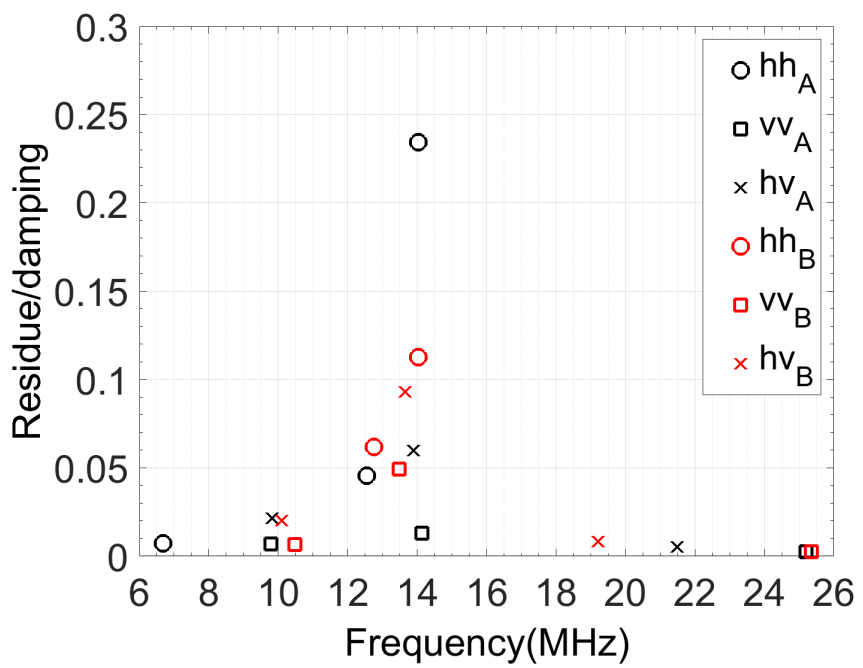

(b)

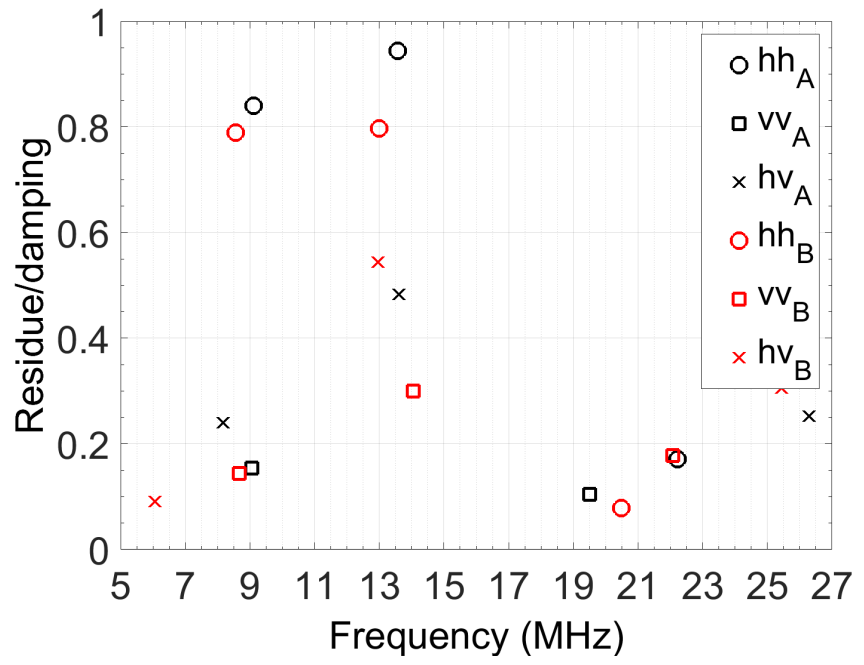

(c)

Figure 11. The mode energy distributions per polarization channel in the backward direction. (A: reference, B: duet model). (a) $T_{1}=225 \mathrm{~ns}$; (b) $T_{1}=347 \mathrm{~ns}$; (c) $T_{1}=113 \mathrm{~ns}$. 


\section{Conclusion}

In general, the high-order modes are of low energy and localized natures which commence adjacent to the end of the specular portion. The estimation of the higher-order modes is more susceptible to onset shift, which in turn adversely affects the modal order of the signature, i.e., level of information. Even more, delaying the onset to avoid specular spill over the transient portion causes the higher modes to be excluded from the signature, i.e., limit the signature modal order. Thus, the higher-order modes are more sensitive to the transient onset shift, and the risk of the modes been estimated incorrectly or even excluded from the signature is high. In general, the forward directions have enhanced the accuracy and consistency of the estimated resonance with a lesser specular return. The results have demonstrated that a generic aircraft model posses distinct modes in the vicinity of $8.89,11.18$ and $23 \mathrm{MHz}$ as expected, where the transient return had duration twice the specular duration of approximately $120 \mathrm{~ns}$. There exist a transient window, almost half its duration, were the modes are most consistent and accurate for the chosen azimuth angle. To further improve the transient return and suppress the specular, we suggest using several modulated pulses of lower resolution, i.e., longer duration, at the frequencies of the target's modes of interest.

\section{Acknowledgements}

This work was supported and funded by The Public Authority of Education and Training, Research Project No (TS-17-14).

\section{Conflicts of Interest}

The author declares no conflicts of interest regarding the publication of this paper.

\section{References}

[1] Cohen, M.N. (1991) An Overview of Radar-Based, Automatic, Noncooperative Target Recognition Techniques. IEEE International Conference on Systems Engineering, Fairborn, 1-3 August 1991, 29-34. https://doi.org/10.1109/ICSYSE.1991.161074

[2] Borden, B. (1994) Problems in Airborne Radar Target Recognition. Inverse Problems, 10, 1009-1022. https://doi.org/10.1088/0266-5611/10/5/002

[3] Sarkar, T.K. and Pereira, O. (1995) Using the Matrix Pencil Method to Estimate the Parameters of a Sum of Complex Exponentials. Antennas and Propagation Magazine, 37, 48-55. https://doi.org/10.1109/74.370583

[4] Hua, Y. and Sarkar, T.K. (1991) On SVD for Estimating Generalized Eigenvalues of Singular Matrix Pencil in Noise. IEEE Transactions on Signal Processing, 39, 892-900. https://doi.org/10.1109/78.80911

[5] Aldhubaib, F. (2019) Binary Stokes Vector Representation of Aircraft in the LowResolution Radar Context. IET Radar, Sonar \& Navigation, 13, 2041-2045. https://doi.org/10.1049/iet-rsn.2019.0066 
[6] Rothwell, E., Kun-Mu, C., Nyquist, D. and Weimin, S. (1987) Frequency Domain E-Pulse Synthesis and Target Discrimination. IEEE Transactions on Antennas and Propagation, 35, 426-434. https://doi.org/10.1109/TAP.1987.1144116

[7] Lee, J.H. and Jeong, S.H. (2012) Performance of Natural Frequency-Based Target Detection in Frequency Domain. Journal of Electromagnetic Waves and Applications, 26, 2426-2437. https://doi.org/10.1080/09205071.2012.735789

[8] Morales, J.D., Blanco, D., Ruiz, D.P. and Carrion, M.C. (2007) Radar-Target Identification via Exponential Extinction-Pulse Synthesis. IEEE Transactions on Antennas and Propagation, 55, 2064-2072. https://doi.org/10.1109/TAP.2007.900259

[9] Stankovic, L., Thayaparan, T. and Dakovic, M. (2006) Signal Decomposition by Using the S-Method with Application to the Analysis of HF Radar Signals in Sea-Clutter. IEEE Transactions on Signal Processing, 54, 4332-4342. https://doi.org/10.1109/TSP.2006.880248

[10] Blanco, D., Ruiz, D.P., Alameda, E. and Carrion, M.C. (2004) An Asymptotically Unbiased E-Pulse-Based Scheme for Radar Target Discrimination. IEEE Transactions on Antennas and Propagation, 52, 1348-1350.

https://doi.org/10.1109/TAP.2004.827251

[11] Aldhubaib, F.F.H. and Shuley, N.V.Z. (2009) Characteristic Polarization States Estimation in an Ultra Wideband Context: A Frequency Approach. IEEE Transactions on Geoscience and Remote Sensing, 47, 2808-2817.

https://doi.org/10.1109/TGRS.2009.2014564

[12] Feko Suit 5. (2003-2005). S.A (Pty) Ltd. 